\title{
Autonomous Robotic Palpation of Soft Tissue using the Modulation of Applied Force
}

\author{
Jelizaveta Konstantinova $^{1}$, Giuseppe Cotugno ${ }^{1}$, Prokar Dasgupta ${ }^{2}$, \\ Kaspar Althoefer $^{1}$, and Thrishantha Nanayakkara ${ }^{1}$
}

\begin{abstract}
Palpation or perception of tactile information from soft tissue organs during minimally invasive surgery is required to improve clinical outcomes. One of the methods of palpation includes examination using the modulation of applied force on the localized area. This paper presents a method of soft tissue autonomous palpation based on the mathematical model obtained from human tactile examination data using modulations of palpation force. Using a second order reactive auto-regressive model of applied force, a robotic probe with spherical indenter was controlled to examine silicone tissue phantoms containing artificial nodules. The results show that the autonomous palpation using the model abstracted from human demonstration can be used not only to detect embedded nodules, but also to enhance the stiffness perception compared to the static indentation of the probe.
\end{abstract}

\section{INTRODUCTION}

$\mathbf{P}$ ALPATION of soft tissues or other viscoelastic environments with a robotic probe is an important field of research for virtual reality and medical applications [1], [2]. Specifically, a number of research studies highlight the importance of reliable tactile feedback during Robot-assisted Minimally Invasive Surgery (RMIS) to improve the clinical outcomes [3].

To implement artificial palpation for surgical applications, the detection of hard abnormalities and measurements obtained by tactile sensing devices should be reliable and repetitive. However, it is difficult to fulfill this requirement due to the variability of conditions introduced by the surgical environment. Variability is caused by the viscoelastic nature of soft tissue as well as by external factors, such as movement of internal organs and flows of bodily fluids.

Manual palpation of soft tissue is performed by expert physicians and surgeons that apply several examination techniques. Namely, they can be divided into three groups: global

This work was supported by National Institute for Health Research (NIHR) Biomedical Research Centre based at Guy's and St Thomas' NHS Foundation Trust and King's College London and Vattikuti Foundation.

${ }^{1}$ J. Konstantinova, G. Cotugno, T. Nanayakkara and K. Althoefer are with the Department of Informatics, King's College London, Strand, London WC2R 2LS, U.K. (e-mail: jelizaveta.konstantinova, giuseppe.cotugno, kaspar.althoefer, thrish.anthaekcl.ac.uk)

${ }^{2} \mathrm{P}$. Dasgupta is with the MRC Centre for Transplantation, DTIMB and NIHR BRC, King's College London, Guy's Hospital, London SE1 9RT,U.K. (e-mail: prokarurol@gmail.com)

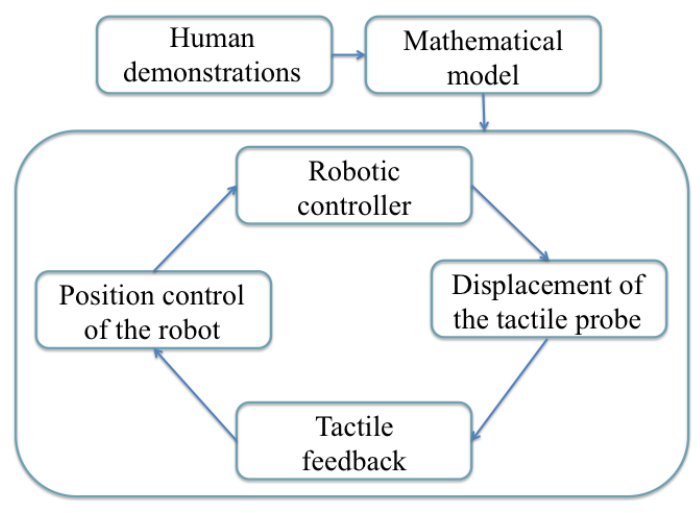

Fig. 1. Schematic representation of autonomous palpation based on human demonstrations.

tissue examination, local tissue examination and the modulation of applied force [4], [5]. Global palpation is required to evaluate the general condition of the organ, as well as to highlight the possible regions of interest that might contain abnormalities. Further on, the local palpation techniques are used to determine the shape and depth of the stiffer region. It is performed by sliding, tapping and vibration of the localized region. The third technique that relies on the modulation of force and corresponds to the average intentional pressure applied to an organ. The pressure can be light or deep, with light used mainly for global scanning and not exceeding 2 $\mathrm{cm}$. Deep palpation is generally used for local examination and is about 4 to $6 \mathrm{~mm}$ deep. The work presented in these studies focuses on deep palpation of the localized area that might contain an abnormality.

There is a broad variety of tactile devices that are developed for surgical purposes, and RMIS specifically [6][8]. In particular, the development of these devices focuses on reduction of their size to fit through a trocar port with the diameter that varies from 5 to $12 \mathrm{~mm}$ [9]. In addition, devices should be either sterilisable, or disposable. The main progress in the development of such devices is made in terms of the mechanical design and measurement principle. There is a good range of tactile devices capable to detect accurate tactile information during static indentation-based palpation. 
Their functionality involves controlled displacement of the probe down in the soft tissue. However, in order to perform efficient tactile examination, it is required to scan the whole surface of an organ in a dynamic way. There are several examples of tactile devices that work based on the principle of active force modulations, e.g. vibrations that are based on resonance frequency methods. For instance, the use of massspring mechanism and linear variable differential transducers in [10], [11] measure viscous and elastic properties of a soft medium due to a shift in the resonance frequency.

The previous studies conducted by authors [12], [13] highlight the importance of an appropriate probing behavior applied during tactile examination. Therefore, there is a strong need to develop not only tactile devices to measure tissue stiffness, but also algorithms and control methods that can be used to enhance tactile perception. It is beneficial to develop devices and algorithms, which could operate in real-time and provide stable measurements for variable environmental conditions. The concept of autonomous robotic palpation is presented in [14]. Hard areas are segmented in a soft environment with the help of elastography and stiffness mapping, and an algorithmic approach to localize stiffer areas is used. Work in [15] describes the autonomous surface recognition using a vibration signal.

This work focuses on understanding the important features of localized tactile exploration that should be taken into account during autonomous or semi-autonomous robotic palpation (Fig. 1). We evaluate the implementation of robotic palpation that is derived on the algorithm outlined from human demonstrations. The palpation is performed on a predefined area on the silicone tissue phantom containing a hard inclusion. Further on, we evaluate the performance of the algorithm comparing it with the indentation-based palpation.

Further on, Section II describes the studies of human palpation and Section III outlines the model; in Section IV the force modulation strategies are implemented using robotic palpation. Section V presents the evaluation of results, followed by Conclusions in Section VI.

\section{Studies of Human Palpation Pattern}

The experimental studies and modeling of human palpation that are used in this work are thoroughly described in [16]. The aim of palpation studies was to explore the way humans use force modulations to explore appointed areas of inhomogeneous environment. Further on, it was required to understand whether there is a generic mathematical model that describes these modulations.

In total, 350 trials were recorded from ten subjects performing palpation on seven areas of silicone phantoms ( 5 trials for each area). In order to stimulate the natural exploration capability during palpation experiments, subjects were asked to estimate the size and depth of the nodule. The appointed area for palpation was covered with an opaque film and was 10 $\mathrm{mm}^{2}$ in size (Fig. 2). The palpation areas on the soft silicone (900 mPa $\times$ s viscosity) had six hard embedded nodules (4000 $\mathrm{mPa} \times \mathrm{s}$ viscosity) and one location without a nodule. The

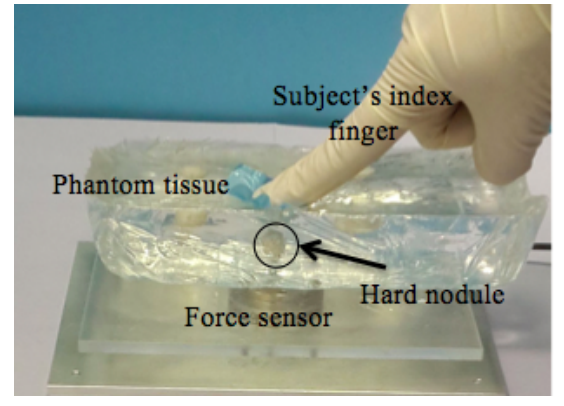

Fig. 2. Experimental setting used for studies of local palpation behavior.

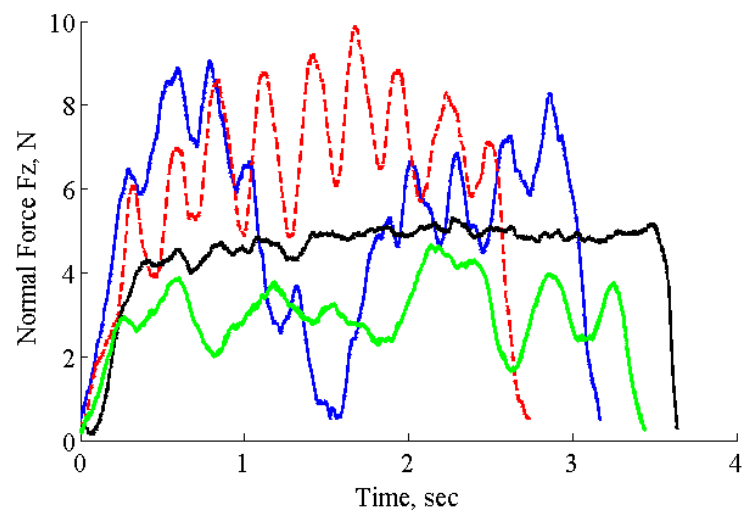

Fig. 3. Sample profiles of normal forces for different subjects.

stiffness ratio between hard nodules and silicone corresponds to the natural difference between a tumor and a healthy tissue [17]. Hard nodules, embedded $5 \mathrm{~mm}$ in depth, had a size comparable to the cancer of stage T1 [18] $(3,6,9,12$ and 15 $\mathrm{mm})$. This type of cancer is very difficult to detect. However, as at this stage the tumor is not widely spread and it can be cured relatively easy.

The applied force was measured by a six-dimensional force and torque sensor (Mini 40, ATI industrial automation) that was placed under the support plate for the silicone phantom. The phantom was fixed on the support plate and any motion or sliding was avoided.

\section{Modeling of Force Modulation}

The mathematical and statistical analysis [16] has shown that humans have a certain pattern for force modulation while palpating an appointed area of interest. Our studies have shown two correlated behaviors of force that are applied during tactile exploration of a localized area. Humans use a threedimensional force to apply pressure during deep palpation $F_{x}, F_{y}$ are lateral forces that coincide with the surface plane of the silicone phantom, and $F_{z}$ is a normal force, orthogonal to the phantom. In particular, the lateral force is applied at a certain vibration frequency, and it is combined with the normal force that can be fitted into a mathematical model. It was found that all subjects apply a similar frequency bandwidth of lateral force with the mean value of $1.8 \pm 0.66 \mathrm{~Hz}$. This value does 
not depend on the size of the nodule and might be related to the physiological constraints of the ligaments of the human hand [19]. The behavior of the normal force also was found to be independent from the size of the nodule, and was modeled using a second order reactive model:

$$
X_{t}=c+\sum_{i=0}^{n} a_{i} X_{t-n}+\varepsilon_{t}
$$

where $c$ is a constant, $a_{i}$ are the autoregression coefficients, $\varepsilon_{t}$ is a white noise, $X_{t}$ is the stress output, $t$ is the sampling step, and $n$ is the model order. In our case the constant $c$ is equal to zero. The coefficients were estimated as: $a_{0}=1, a_{1}=$ $-1.706, a_{2}=0.721$. Samples profiles of normal forces applied during palpation are shown in Fig. 3.

\section{RoBotic PALPATION}

The robotic implementation realizes two simultaneous correlated behaviors derived from human demonstrations. The aim of the experiments is not just to mimic the human behavior, but also to understand whether this pattern can be used in autonomous or semi-autonomous robotic palpation. In other words, whether the modulation of applied force can enhance the perception of viscoelastic environment compared to simple static probing. In addition, we are interested in the perception of small nodules that were sometimes missed by human subjects.

\section{A. Experimental Setup}

In order to implement robotic palpation, a tactile probe with spherical indenter is used. The diameter of indenter is $8 \mathrm{~mm}$. This diameter is chosen in order not to damage the soft material, and it is also comparable to the size of human finger. To perceive forces from the interaction with the phantom tissue, a 6 DoF force and torque sensor is used (NANO17, ATI industrial automation, normal force resolution $1 / 320 \mathrm{~N})$. The probe is attached to the robotic arm Fanuc M$6 \mathrm{iB}$ with R-J3iB controller. This robot arm has $6 \mathrm{DoF}$ and \pm $0.08 \mathrm{~mm}$ repeatability of the motion. The control of the robot is implemented in position space using perceived force as a control signal (Fig. 1). The experimental setting is shown in Fig. 4.

The palpation studies are focused on the exploring of force modulations on the localized area only. Therefore, the tactile probe was positioned above the region of interests before the start of palpation. In order to replicate the conditions of deep palpation and not to break the phantom tissue [20], the threshold of the indentation depth of the probe was set to 6 $\mathrm{mm}$. Moreover, the applied indentation threshold is required to keep the linear elastic limit of the phantom tissue in order to perform stiffness calculation, as described later.

\section{B. Design of the Experiments}

The design of the experiments is based on the following principles:

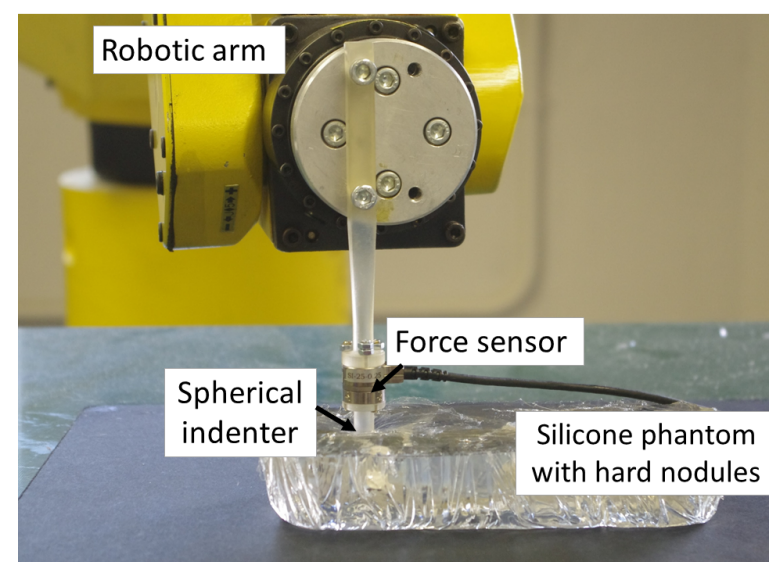

Fig. 4. Experimental setup to validate autonomous palpation based on force modulation strategy

1) To obtain stiffness from the phantom tissue using autonomous palpation, based on the outlined mathematical model;

2) To obtain stiffness from passive or indentation-based palpation, such as described in [21]. For this palpation type the stiffness is calculated after the probe is indented into the material.

3) To compare the stiffness perception from the same locations and evaluate whether the proposed model can be used for robotic applications.

To calculate the stiffness of the perceived environment from the force measurements, the Young's modulus is used. According to literature [22], the Young's modulus of soft tissue for a spherical indenter and an indentation depth, not exceeding the linear elastic limit, can be calculated using the following formula:

$$
E=\frac{3 f(1+v)}{8 d_{i n} \sqrt{r d_{i n}}}
$$

where, $E$ is the Young's modulus and $v$ is the Poisson's ratio of the material, $f$ is the normal interaction force with the tissue, $r$ is the radius of the indenter $(8 \mathrm{~mm})$, and $d_{i n}$ is the indentation depth. Poisson's ratio for the assumed incompressible soft tissue is 0.5 .

In order to preserve the consistency of robotic experiments with human demonstrations, the same silicone phantoms were used. Hard nodules of different sizes (3, 6, 9, 12 and $15 \mathrm{~mm}$ ), as well as a homogeneous empty area in the silicone were used for testing. In such a way, the robotic palpation was performed on the nodules that can be easily detected by humans (12 and $15 \mathrm{~mm}$ ), as well as on the nodules that can be missed during human palpation ( 3 and $6 \mathrm{~mm}$ ). To asses the feasibility of nodule detection, the stiffness of the homogeneous area of the silicone was measured as well. Five trials of robotic palpation based on human demonstrations were performed for each area - empty location and five nodules.

In the second stage of the experimental studies, the indentation-based palpation is performed. To measure the stiff- 


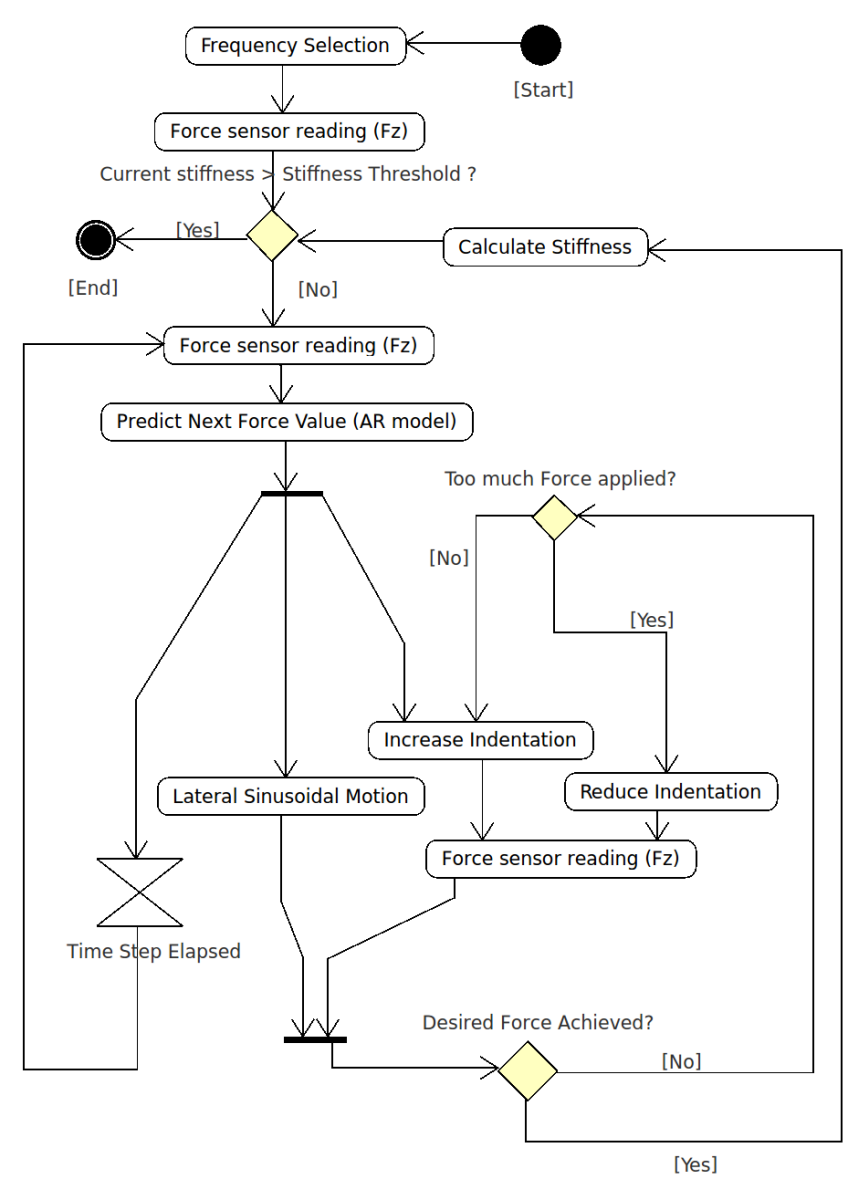

Fig. 5. Flowchart of the algorithm designed for robotic palpation.

ness, the probe is indented $5 \mathrm{~mm}$ down into the appointed area on the silicone phantom. After the indentation, the interaction force with the phantom is measured, and the corresponding stiffness is calculated. Further on, the obtained measurements are used to evaluate the feasibility of the proposed autonomous palpation.

\section{Control Algorithm}

This section describes the design of the control algorithm for autonomous palpation. The flowchart of the autonomous palpation algorithm is displayed in Fig. 5. The initialization of the system is implemented at the first steps of the algorithm. Initially, the frequency of the whole system is selected based on the empirical evaluation and set to $120 \mathrm{~ms}$. This is the time allowed to achieve the required force given by the autoregressive predictor. Another constituent parameter of the system is the selection of stiffness threshold. This is the value obtained during indentation-based stiffness measurement and is used as a termination and assessment criteria for the system.

The first force readings are used to initialize the second order AR model. They are recorded for two indentation steps, each $0.25 \mathrm{~mm}$ deep. The initialization stage is crucial for correct execution of the palpation, as AR model requires two input parameters. Subsequently, the system enters the palpation loop. Two types of motions are performed iterative manner: lateral sinusoidal motion and normal motion. Lateral movement creates the sinusoidal modulation of lateral force according to human studies, with the frequency $1.8 \mathrm{~Hz}$. The range of the lateral motion is empirically set to $2 \mathrm{~mm}$ in order to correspond to the desired frequency. Normal motion is generated according to the desired force that is defined by the AR model.

In each iteration, the system reads force measurements according to the current position of the probe; predicts a desired force, using the AR model; and translates the force into position. This translation is performed via incremental or decremental indentations of $0.25 \mathrm{~mm}$ based on the errors from the sensor with the tolerance of $0.01 \mathrm{~N}$. If the desired force is not achieved within the given time limit (120 ms), current force value is fed into AR model for the next prediction. At the end of each iteration the stiffness is evaluated according to Eq. 2. As soon as the value (threshold set by indentation-based palpation) is achieved, the autonomous palpation continues for 30 seconds to observe the variation of the stiffness dynamics.

\section{VALidation of Results For Robotic Palpation}

This section evaluates and compares the stiffness measurement results obtained from robotic palpation. The stiffness measurements obtained from passive indentation-based palpation are shown in Fig. 6 as red dotted lines. This value of stiffness is used as a criterion to evaluate the feasibility of autonomous palpation.

The stiffness measurement obtained from autonomous palpation based on human demonstrations is shown in blue solid lines on the same figure (Fig. 6). The measured stiffness demonstrates the modulations that are caused by the variable dynamics of the probe, as well as the motion of the material. These dynamic modulations fluctuate around the indentationbased stiffness value. It can be observed that the proposed palpation motion generates a dynamic gain that is likely to enhance the perception of stiffness.

To explore the meaning of such response, we study variance and magnitude of the stiffness, as well as compare it with the stiffness obtained during static palpation. The first parameter variance, denotes the spread of the stiffness value relatively to the mean value. It also characterizes the temporal modulation of stiffness during autonomous palpation. The value of mean variance corresponding to each nodule location is shown on Fig. 7 a). To evaluate whether there is a significant difference between the variance for different palpation locations, a nonparametric Wilcox rank test was performed. This test was chosen because of the small number of samples. The statistical test shows that there is a significant difference for the location with no nodule and palpation areas with nodules of 12 and 15 $\mathrm{mm}$ in diameter $(p<0.05)$. Thus, the variance of stiffness caused by autonomous palpation demonstrates that it can enhance the perception for relatively big size nodules.

The second parameter is the mean magnitude of the stiffness calculated relatively to the static palpation. The evaluation of the magnitude demonstrates weather the autonomous palpation 

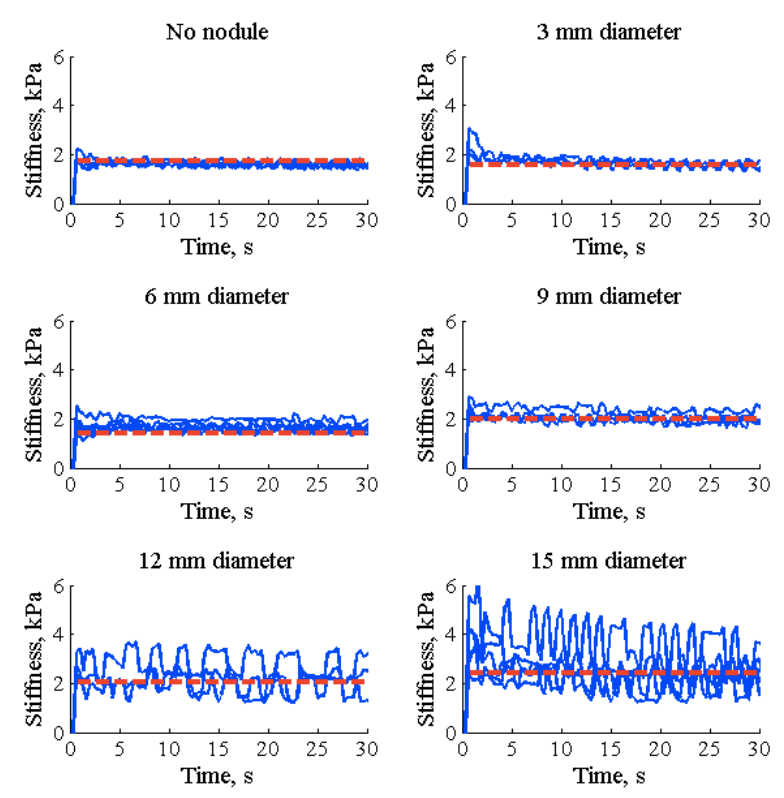

Fig. 6. Stiffness measurements for indentation based measurements (red dotted line) and autonomous palpation based on human demonstrations (blue solid line) for silicone with no nodule, nodules of 3, 6, 9, 12 and $15 \mathrm{~mm}$.

based on human demonstrations can enhance the perception of stiffness from non-homogeneous areas. These differences in magnitude for all palpation areas are displayed in Fig. 7b). It can be observed that the mean magnitude for the nodules obtained during autonomous palpation exceeds the stiffness measured during static palpation. However, this positive difference is not observed for the palpation of the homogeneous area with no nodule. This means that the proposed palpation model can be used to enhance the stiffness perception from non-homogeneous environment.
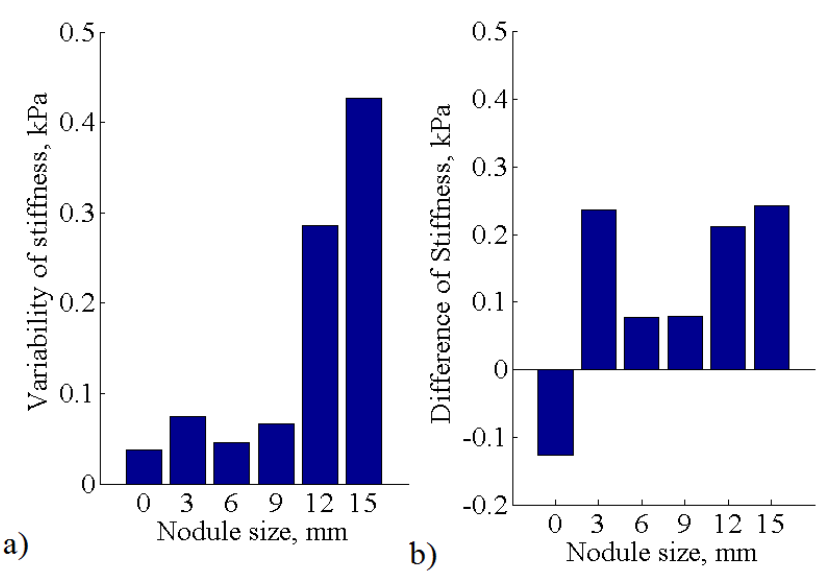

Fig. 7. a) Variability of stiffness measurements for autonomous palpation, and b) Difference of stiffness for autonomous palpation and indentation based measurement, for silicone with no nodule, nodules of 3, 6, 9, 12 and $15 \mathrm{~mm}$
To visualize the effect of autonomous palpation, Fig. 8 presents a combined graph of the variance and the mean magnitude relatively to static stiffness plotted against each other. This graph clearly shows the effect of the proposed behavior. The zero point on the graph denotes the value of the static stiffness for all nodules. If the point on the graph is close to zero then there is little difference with the indentation-based palpation. In case the point is below the zero line, the performance of the autonomous palpation is considered not efficient for the given environment. According to Wilcox rank test there is a significant difference $(p<0.05)$ between measurements displayed below and above the zero. High variance of stiffness and higher magnitude compared to the static palpation denotes the effectiveness of autonomous palpation compared to the indentation-based measurements. This can be observed for all cases of palpation of the appointed area containing nodules.

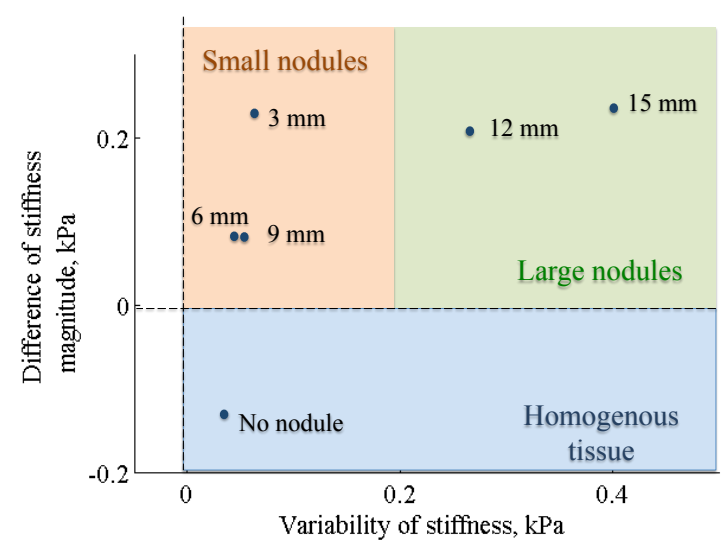

Fig. 8. Variability and difference of stiffness of hard nodules and soft environment for robotic palpation for different nodule sizes.

\section{CONCLUSIONS}

This work presents a concept of autonomous palpation that is based on human demonstrations. It was shown that the human model of force modulations during palpation can be successfully implemented for robotic application. Moreover, such behavior enhances the perception of stiffness.

In the context of this work, it is worth to note that to implement this approach for practical applications it is required to take into account the other challenges related to robotic palpation. For instance, it is required to take into account the curvatures of organ shapes during tool control; either by measuring both force and indentation [23], or by using virtual representation of the environment [20].

Our studies presented in this work discuss the autonomous palpation of a small appointed area. However, it is required to take into account the complexity of the palpation environment, as well as the safety issues associated with surgery. This makes tele-manipulated remote tactile examination the most suitable for global palpation. Therefore, it can be used in combination 
with autonomous palpation for small areas only to enhance the quality of examination.

\section{ACKNOWLEDGMENT}

The research has received funding the National Institute for Health Research (NIHR) Biomedical Research Centre at Guy's and St Thomas' NHS Foundation Trust and King's College London, Vattikuti Foundation, and the MRC Centre for Transplantation, KCL, KHP. The views expressed are those of the authors and not necessarily those of the NHS, the NIHR or the Department of Health.

\section{REFERENCES}

[1] A. E. Saddik, "The Potential of Haptics Technologies," IEEE Instrumentation and Measurement Magazine, no. February, pp. 10-17, 2007.

[2] A. M. Okamura, "Haptic Feedback in Robot-assisted Minimally Invasive Surgery," Current Opinion in Urology, vol. 19, pp. 102-7, Jan. 2009.

[3] A. Talasaz and R. V. Patel, "Integration of force reflection with tactile sensing for minimally invasive robotics-assisted tumor localization," IEEE Transactions on Haptics, vol. 6, no. 2, pp. 217-228, 2013.

[4] N. Wang, G. J. Gerling, R. M. Childress, and M. L. Martin, "Quantifying Palpation Techniques in Relation to Performance in a Clinical Prostate Exam," IEEE Trans. on Information Technology in Biomedicine: a Publication of the IEEE Engineering in Medicine and Biology Society, vol. 14, pp. 1088-97, July 2010.

[5] K. J. Saunders, C. A. Pilgrim, and H. S. Pennypacker, "Increased Proficiency of Search in Breast Self-examination," Cancer, vol. 58, pp. 2531-7, Dec. 1986.

[6] M. Ohka, T. Matsunaga, Y. Nojima, D. Noda, and T. Hattori, "Basic experiments of three-axis tactile sensor using optical flow," in 2012 IEEE International Conference on Robotics and Automation, pp. 1404-1409, Ieee, May 2012.

[7] H. Xie, H. Liu, S. Luo, L. D. Seneviratne, and K. Althoefer, "Fiber Optics Tactile Array Probe for Tissue Palpation during Minimally Invasive Surgery," in IEEE/RSJ International Conference on Intelligent Robots and Systems, pp. 2539-2544, 2013.

[8] D. Greenwald, C. G. L. Cao, and E. W. Bushnell, "Haptic Detection of Artificial Tumors by Hand and with a Tool in a MIS Environment," IEEE Transactions on Haptics, vol. 5, no. 2, pp. 131-138, 2012.

[9] D. Mckay and G. Blake, "Optimum Incision Length for Port Insertion in Laparoscopic Surgery," The Annals of The Royal College of Surgeons of England, vol. 88, no. 1, p. 78, 2007.

[10] M. Jia, J. W. Zu, and A. Hariri, "A New Tissue Resonator Indenter Device and Reliability Study," Sensors, vol. 11, pp. 1212-1228, Jan. 2011.

[11] M. H. Araghi and S. P. Salisbury, "A feedback Based Dynamic Instrument for Measuring Mechanical Properties of Soft Tissues for Minimally-Invasive surgery," in Smart Materials and Structures, no. October, pp. 59-69, 2009.

[12] J. Konstantinova, M. Li, V. Aminzadeh, K. Althoefer, P. Dasgupta, and T. Nanayakkara, "Evaluating Manual Palpation Trajectory Patterns in Tele-Manipulation for Soft Tissue Examination," in IEEE Systems Men and Cybernetics, pp. 4190-4195, 2013.

[13] J. Konstantinova, M. Li, G. Mehra, P. Dasgupta, K. Althoefer, and T. Nanayakkara, "Behavioral characteristics of manual palpation to localize hard nodules in soft tissues," IEEE Transactions on Biomedical Engineering, vol. 61, no. 6, pp. 1651-1659, 2014.

[14] K. A. Nichols and A. M. Okamura, "Autonomous Robotic Palpation: Machine Learning Techniques to Identify Hard Inclusions in Soft Tissues," in IEEE International conference on Robotics and Automation ICRA, pp. 4369-4374, 2013.

[15] J. M. Romano and K. J. Kuchenbecker, "Methods for robotic toolmediated haptic surface recognition," in 2014 IEEE Haptics Symposium (HAPTICS), pp. 49-56, 2014.

[16] J. Konstantinova, Tactile probing strategies for soft tissue examination. $\mathrm{PhD}$ thesis, 2015.

[17] "Elastic Moduli of Breast and Prostate Tissue under Compression," Ultrasonic Imaging, vol. 20, pp. 260-274, 1998.
[18] W. A. Woodward, E. A. Strom, S. L. Tucker, M. D. McNeese, G. H Perkins, N. R. Schechter, S. E. Singletary, R. L. Theriault, G. N Hortobagyi, K. K. Hunt, and T. A. Buchholz, "Changes in the 2003 American Joint Committee on Cancer staging for breast cancer dramatically affect stage-specific survival.," Journal of Clinical Oncology, vol. 21, pp. 3244-8, Sept. 2003.

[19] R. W. Haines, "The mechanism of rotation at the first carpo-metacarpal joint," Journal of anatomy, vol. 78, pp. 44-46, 1944.

[20] M. Li, J. Konstantinova, E. L. Secco, A. Jiang, H. Liu, T. Nanayakkara, L. D. Seneviratne, P. Dasgupta, K. Althoefer, and H. A. Wurdemann, "Using visual cues to enhance haptic feedback for palpation on virtual model of soft tissue," Medical \& Biological Engineering \& Computing, pp. 1-10, 2015.

[21] H. Liu, D. P. Noonan, B. J. Challacombe, P. Dasgupta, L. D. Seneviratne, and K. Althoefer, "Rolling Mechanical Imaging for Tissue Abnormality Localization during Minimally Invasive Surgery," IEEE Trans. on Biomedical Engineering, vol. 57, pp. 404-14, Feb. 2010.

[22] E. H. Lee and J. R. M. Radok, "Contact problem for viscoelastic bodies," Journal of Applied Mechanics, vol. 27, no. 3, pp. 438-444, 1960.

[23] I. B. Wanninayake, L. D. Seneviratne, and K. Althoefer, "Novel indentation depth measuring system for stiffness characterization in soft tissue palpation," 2012 IEEE International Conference on Robotics and Automation, pp. 4648-4653, May 2012. 